Research Article

\title{
Recall and Interviewer Bias in Parental Report of Pediatric Exposure to Aromatic Plant Ingredients in Personal Care Products: Development and Validation of a More Accurate Approach
}

\author{
Jessie Hawkins $\mathbb{D}$, Christy Hires $\mathbb{D}$, Elizabeth Dunne, and Lindsey Keenan \\ Franklin Health Research Foundation, Franklin, TN 37067, USA \\ Correspondence should be addressed to Jessie Hawkins; j.hawkins@franklinhealth.org
}

Received 23 March 2021; Revised 7 July 2021; Accepted 16 August 2021; Published 24 August 2021

Academic Editor: Chunrong Jia

Copyright (C) 2021 Jessie Hawkins et al. This is an open access article distributed under the Creative Commons Attribution License, which permits unrestricted use, distribution, and reproduction in any medium, provided the original work is properly cited.

\begin{abstract}
Lavender and tea tree essential oils are traditionally considered to be mild, gentle, and safe for pediatric populations and are ubiquitous in personal care products. Recent case reports have proposed a potential association between exposure to these ingredients and endocrine disruption, but these reports contain misclassification bias. The purpose of this study is to develop a reliable and valid measurement instrument for the accurate classification of exposure to aromatic plant ingredients in personal care products to be used in epidemiological studies. This study tested the Aromatic Plant Ingredients and Child Health Survey (APICHS) for validity and reliability, contrasting it with the current approach used in clinician's offices. The APICHS was found to have exceptional sensitivity and specificity (100\% and $92.86 \%$, respectively) with a positive predictive value of $97.22 \%$, far exceeding the sensitivity and specificity of the method currently in use. The APICHS is a valid, reliable tool for accurate classification of exposure to aromatic plant ingredients in personal care products and should be used for the avoidance of misclassification.
\end{abstract}

\section{Introduction}

Lavender and tea tree essential oils are among the most common ingredients in personal care products, especially for children $[1,2]$. The oils are traditionally considered to be mild, gentle, and safe for pediatric populations [3-5]. Recent sales of personal care products confirm that these are desirable ingredients in household products for families [6]. However, recent case reports have proposed that these two oils are associated with endocrine disruption in children [7]. Due to a lack of epidemiological evidence, experts have advised caution until the proposed link between ingredients and the rare outcome of prepubertal gynecomastia can be investigated. Parents of young children have expressed concern over the lack of clarity on the potential risk posed by these oils, further confirming the urgent need for this epidemiological research.
Because lavender essential oil and tea tree essential oil are ubiquitous in personal care products, identifying and properly classifying exposure status presents a significant challenge. This challenge poses a barrier to the epidemiological research required to scientifically assess the claims that these essential oils act as endocrine disrupting agents in the developing human body.

Lavender and tea tree essential oils do not leave traces at any detectable level within the human body $[8,9]$. Key chemicals in these plant extracts have half-lives as short as 14-18 minutes, rendering a laboratory test for past exposure infeasible $[10,11]$. To identify historical exposures to these ingredients, many clinicians simply ask parents during an office visit whether or not their child has used any personal care products which contain lavender or tea tree essential oils. This recall question is the only tool utilized to classify the child as exposed or unexposed. 
This approach lacks the scientific rigor required to classify exposure status of a child, due to its failure to address information bias, specifically recall bias and interviewer bias, ultimately resulting in misclassification bias $[12,13]$. Parents are rarely able to recite the full list of ingredients in all of their child's skincare products, and the stress of a specialist visit with a young child inhibits recall ability, particularly when a diagnosed illness is involved $[14,15]$.

Similarly, clinicians are not unbiased data collectors; their attitude about the ingredient in question is conveyed to the parent through nonverbal communication. This can result in the Hawthorne effect, when parents answer as they believe they are being led to answer rather than with factual information [16]. Additionally, interviewer bias (when clinicians obtain skewed data in support of preconceived notions) is introduced when clinicians ask cases about exposure status but fail to question noncases about potential exposure [17-19]. Ultimately, this approach produces data which reflects clinician perceptions rather than reality.

In epidemiological studies, misclassification bias, the failure to accurately classify exposed and unexposed patients, distorts research findings. Differential misclassification is particularly problematic because overclassification of exposed cases biases the risk, odds, or rate ratios away from the null [20]. This inaccuracy in data collection leads to an artificially inflated sense of risk.

Because some chemical research has proposed a relationship between these two ingredients and endocrine disruption in children, a valid and reliable method of classifying patients as exposed and unexposed is urgently required [7]. Misclassification has already occurred in the scientific literature regarding these ingredients, with at least one highly publicized case series failing to accurately classify exposures [21]. Misclassification of exposures results in inaccurate reports in the literature and ultimately, the prolonging of key public health issues. Until exposure status can be accurately classified, the epidemiological research studies necessary to investigate this proposed link cannot be conducted.

1.1. Objective. The primary purpose of this study is to evaluate the Aromatic Plant Ingredients and Child Health Survey (APICHS) for validity and reliability as a measurement instrument. The purpose of this instrument is to accurately classify children who were exposed to lavender or tea tree essential oil through personal care products and household exposures between the ages of 2 and 15 years old. The secondary objective of this study is to evaluate the prior approach of parental recall for validity and reliability and determine which approach has greater positive predictive value. This study is reported using the STARD checklist for diagnostic accuracy studies.

\section{Methods}

2.1. Study Design. This is a prospective study evaluating two methods of measuring pediatric exposure to aromatic ingredients in personal care and household products.
2.2. Participants. The population for this study consists of parents of children ages $2-15$ years old who live in the United States. Parents were recruited from across the country. Informed consent and instructions for the survey were administered online. After signing the informed consent, the parents completed the health outcome survey and identified personal care products to which their child had been exposed. The study was approved by an independent IRB prior to recruiting the first participants.

2.3. Sample Size. A power analysis was conducted using $\mathrm{G}^{*}$ Power 3.9.1.6 to determine the minimum sample size required to identify a strong correlation ( 0.8 or higher). Considering 0.05 as statistically significant, with $90 \%$ power, a minimum of 23 participants were required to identify a correlation of 0.8 or higher. To account for dropouts and to allow for subgroup analyses, the minimum sample was increased by $50 \%$ to 35 .

2.4. Test Methods. Two methods of measuring exposure were used for this study. The Aromatic Plant Ingredients and Child Health Survey (APICHS) was used, as well as the current approach of a single yes/no question, "Do products used on this child contain (lavender/tea tree) essential oil?" The APICHS was developed specifically for use with this population to improve classification of exposures for both diagnostic and epidemiological research purposes. To ensure that the APICHS did not influence responses to the yes/no question, the APICHS was given after parents responded to the current approach.

2.5. Recall Bias. Recall bias is a considerable issue with retrospective measurement and self-reporting, so the APICHS was developed with the understanding that it is impractical to expect parents to recall the full ingredient lists of all items their children have used over the last 2-15 years. Rather than ask parents about ingredients, the instrument uses visual prompts to collect exposure data based on market-ready product usage. Studies have shown that visual prompts are highly effective in promoting memory recall [22-24]. The APICHS uses visual cues with introductory texts to help parents recall which products their child has used.

2.6. APICHS Development. The survey contains two sections. Section one includes demographic, health history, health outcomes, and essential oil home use questions. These questions are categorical or dichotomous variables. Section two contains personal care product identification with over 400 images of personal care products, each which either contain one of the essential oils in question or are marketed in such a way to imply that they contain one of the essential oils in question.

To ensure a fully representative population of products which may contain lavender or tea tree essential oils, a comprehensive search across web-based stores, 
manufacturers, and brick-and-mortar stores was conducted. In addition to individual retailers and web searches, databases such as the Environmental Working Group's Skin Deep database were searched, as well as mass market retailers such as Amazon.com. To capture discontinued products, Internet archives were used as well. When there was more than one formulation for a product, both products were included, along with the visual identifiers. In total, ingredient lists for approximately 4,500 products were evaluated for potential inclusion. If a product implied that it had lavender essential oil, tea tree essential oil, lavender extract, or fragrances, it was included in the screening.

Each product was classified as a wash-off personal care product (i.e., shampoo or body wash), a leave-on product (i.e., lotion, hair styling gel), or an environmental household exposure (i.e., laundry soap). Products were classified as containing pure lavender essential oil, pure tea tree essential oil, primary chemicals found in lavender essential oil (i.e., linalool), primary chemicals found in tea tree oil, or none of the ingredients in question. These allowed for the development of a codebook which classified each suspected exposure as a true exposure or not. The codebook was based on the product's ingredient list, which is subject to FDA cosmetic regulations [25].

2.7. Analysis. Because direct observation is not possible and there is no existing measurement with which to compare, validity and reliability were evaluated through test-retest with one week between responses. Because one of the greatest threats to retrospective data collection is recall bias, test-retest reliability serves as an optimal measure to evaluate the appropriateness of this instrument for the purpose of diagnostics and epidemiological research on the risks and safety of exposures to ingredients in personal care products.

Using the index test, total exposure scores were calculated at point 1 and point 2 for both lavender and tea tree oil. This provided a figure for the total number of different exposures to each of these ingredients. The mean difference in exposure scores between the two timepoints was computed to evaluate the difference between testing and retesting [26]. These continuous outcomes were evaluated using Pearson's correlation coefficient as well as Lin's concordance correlation coefficient with a $z$-transformation and a bias correction factor [27]. A scatterplot of exposure scores with a line of linear fit was created to produce a visual assessment of the relationship between testing points and a Bland-Altman plot was constructed to identify the limits of agreement between the two timepoints with a 95\% confidence interval [28].

To assess diagnostic accuracy, the continuous variable of total exposure was converted to a dichotomous variable of exposed and unexposed. Additionally, the current yes/no single-question approach was assessed. For each measurement test, a Kappa coefficient of agreement was calculated. Diagnostic utility was evaluated via sensitivity, specificity, and positive and negative predictive values with baseline scores as the reference.

\section{Results}

3.1. Participant Flow. Of the 70 parents of children aged 2-15 who were recruited to participate, 58 completed the first survey. A total of 53 completed the second survey, one week later. A total of 4 participants failed to completely finish one or both surveys, producing a total sample size of 49. Parents spent an average of 14 minutes on the first survey and 11 minutes on the second survey. The mean age of children represented in the survey was $7.63(\mathrm{SD}=3.78)$. There were slightly more females than males (22 or $44.9 \%$ and 27 or $55.1 \%)$, and $43(87.76 \%)$ of the children were white. Household income and parental education were approximately normally distributed.

3.2. Correlation of Total Exposure Values. Lavender exposure scores during the first survey ranged from 0 to 11 products with a mean of $3.20(\mathrm{SD}=2.86)$. Exposure scores during the retest survey also ranged from 0 to 11 products with a mean of 3.39 $(\mathrm{SD}=3.05)$. The scatterplot of scores comparing the two measurements, with a line of linear fit demonstrating high levels of agreement. This was confirmed with Pearson's correlation coefficient, which identified a strong linear correlation between the two measures, $r=0.821, n=49, p=<0.001$.

Tea tree exposure scores were much lower for both surveys. Scores ranged from 0 to 2 on the first test and $0-3$ on the retest. Average scores were $0.27(\mathrm{SD}=0.53)$ on the first test and $0.41(\mathrm{SD}=0.79)$ on the retest. The scatterplot of scores comparing the two measurements, with a line of linear fit demonstrates acceptable agreement, which was confirmed by Pearson's correlation coefficient of .73 $(n=49$, $p=<0.001)$.

3.3. Concurrent Validity. To evaluate the extent to which questionnaire responses one week apart agree on lavender exposure, Lin's concordance correlation coefficient was calculated with a bias transformation factor. Exposure responses from week 1 and week 2 were found to have an acceptable level of concordance.

Linn's concordance correlation coefficient identified agreement between the two scores $\left(\rho_{c}=0.82,95 \%\right.$ confidence limits with $z$-transformation $0.696,0.891)$. See Table 1 .

Lin's concordance correlation coefficient was also calculated with a bias transformation factor for the outcome of tea tree exposure. Exposure responses from week 1 and week 2 were found to have a lower level of concordance. Linn's concordance correlation coefficient identified agreement between the two scores $\left(\rho_{\mathrm{c}}=0.66,95 \%\right.$ confidence limits with $z$-transformation $0.503,0.778)$. See Table 2 .

3.4. Bland-Altman. A Bland-Altman plot also revealed agreement between responses for total lavender essential oil and total tea tree essential oil exposures. For the lavender essential oil outcome, the mean difference in exposure scores was $0.265 \quad(\mathrm{SD}=1.765)$, with Bland-Altman limits of agreement of -3.194 and 3.725 (95\% CI). On average, responses to exposures were remarkably similar (see Figure 1). 
TABle 1: Agreement between lavender exposure measures.

\begin{tabular}{|c|c|c|c|}
\hline Statistical approach & Statistic & 95\% confidence interval & $p$ \\
\hline Lin's concordance correlation coefficient $\rho_{c}$ & 0.815 & $0.696,0.891$ & $<0.001$ \\
\hline Pearson's $r$ & 0.821 & - & $<0.001$ \\
\hline Bias correction factor & 0.993 & - & - \\
\hline Bland-Altman's limits of agreement & $0.265(\mathrm{SD}=1.765)$ & $-3.194,3.725$ & - \\
\hline
\end{tabular}

TABLE 2: Agreement between tea tree exposure measures.

\begin{tabular}{|c|c|c|c|}
\hline Statistical approach & Statistic & 95\% confidence interval & $p$ \\
\hline Lin's concordance correlation coefficient $\rho_{c}$ & 0.662 & $0.503,0.778$ & $<0.001$ \\
\hline Pearson's $r$ & 0.731 & - & $<0.001$ \\
\hline Bias correction factor & 0.906 & - & - \\
\hline Bland-Altman's limits of agreement & $0.143(\mathrm{SD}=0.54)$ & $-0.916,1.201$ & - \\
\hline
\end{tabular}

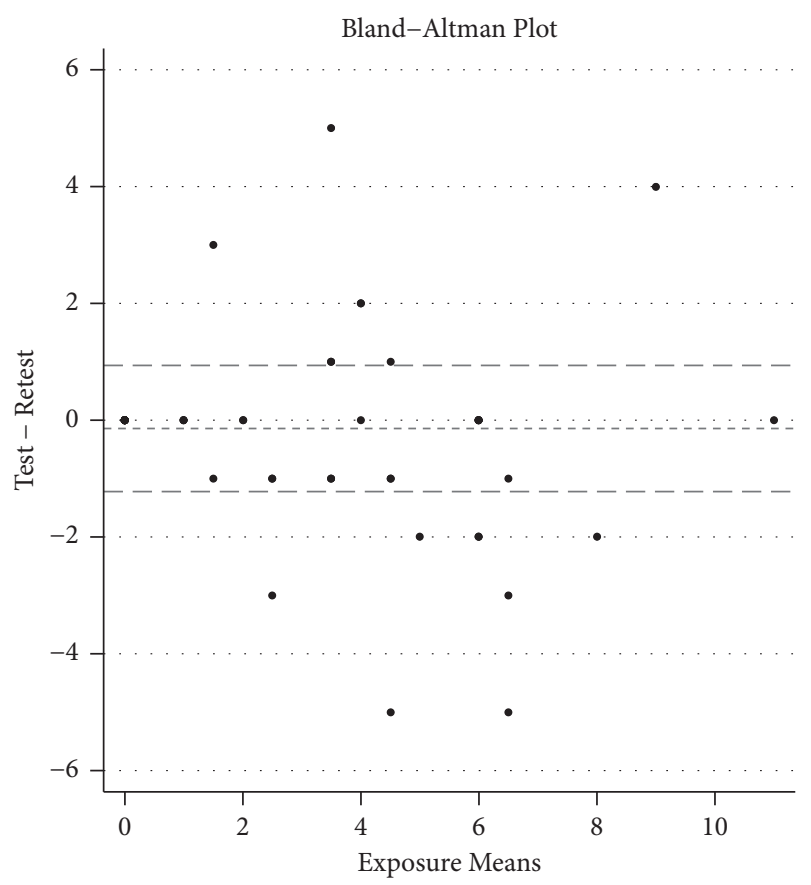

FIGURE 1: Bland-Altman limits of agreement: -3.194 and 3.725 (95\% CI).

For the tea tree essential oil outcome, the mean difference in exposure scores was $0.143(\mathrm{SD}=.540)$, with Bland-Altman limits of agreement of -0.916 and $1.201(95 \%$ CI) (see Figure 2).

3.5. Exposed versus Unexposed. While the continuous outcomes provide utility evaluating the total number of unique exposures, the outcome relevant to most clinicians and epidemiologists is exposure status, regardless of dose. To evaluate the ability of the APICHS method of accurately classifying patients as exposed or unexposed, the variables were converted to dichotomous reflecting complete absence of exposure and any level of exposure. This allowed direct comparison with the current approach of a single yes/no question.

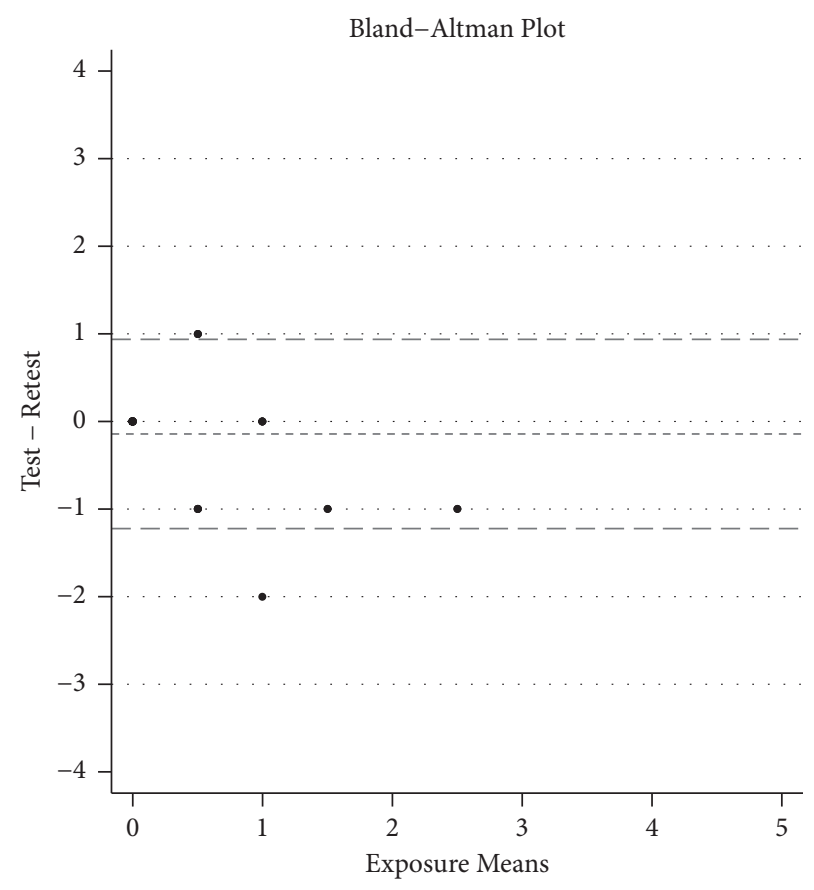

FIGURE 2: Bland-Altman limits of agreement: -0.916 and 1.201 $(95 \% \mathrm{CI})$.

3.6. Kappa Coefficient. Cohen's $\kappa$ was performed to determine the extent of agreement between the measures at the two points in time. For the outcome of lavender exposure, the index test produced almost perfect agreement, $\kappa=0.949$ (95\% CI: $0.850,1.00), p=<0.001$ ), whereas the current approach only produced moderate agreement, $\kappa=.550(95 \%$ CI: 0.306, 0.795), $p=<0.001)$.

For the outcome of tea tree essential oil exposure, both tests produced moderate agreement, $\kappa=0.609$ (95\% CI: $0.380,0.838), \quad p=<0.001 ; \kappa=0.560$ (95\% CI: 0.290 , $0.829), p=<0.001)$. Fewer than 15 total patients reported tea tree exposure via either method, which could account for the similarity in scores for the outcome of tea tree exposure. 
3.7. Diagnostic Utility: Sensitivity and Specificity. To evaluate external validity and diagnostic utility, sensitivity, specificity, positive predictive value, and negative predictive value were evaluated. When evaluating exposure to lavender essential oil, sensitivity and specificity were $100 \%$ and $92.86 \%$, respectively. Positive predictive value was $97.22 \%$ and negative predictive value was $100 \%$, with the model correctly classifying $97.96 \%$ of participants. The index test had a $0 \%$ false negative rate and a $2.78 \%$ false positive rate.

These outcomes were far superior to the current method, which produced sensitivity and specificity of $82 \%$ and $75 \%$, respectively. Positive predictive value was $87.10 \%$ and negative predictive value was $66.67 \%$, with the model correctly classifying only $79.6 \%$ of patients. The current approach had a $33.3 \%$ false negative rate and a $12.9 \%$ false positive rate.

Similar results were identified on the tea tree exposure analysis. Sensitivity was found to be $61.54 \%$, with $91.67 \%$ specificity. Positive predictive value was $73.72 \%$ and negative predictive was $86.84 \%$, with the model correctly classifying $83.67 \%$ of patients. In contrast the current approach has $73.68 \%$ sensitivity and $86.67 \%$ specificity, with a $77.78 \%$ positive predictive value and $83.87 \%$ negative predictive value, correctly classifying $81.63 \%$ of exposures.

\section{Discussion}

4.1. Limitations. One strength of this approach is that the tool utilizes images, allowing for its use with non-English speaking populations. However, due to variations in consumer product availability, the tool is limited to the United States markets.

Additionally, because new products are introduced to the market on a regular basis, and product formulations are frequently updated, the tool must also be updated at a minimum annually. Given the poor predictive value of the current approach, exposures should be classified using the most accurate measure available, especially given the recent attempts to assign causation based on misclassified data. Inaccurate assignment of causation fails the patient by creating unnecessary burdens and fears of personal care products and also fails to identify the actual cause of endocrine disruption, leaving the patient vulnerable to increased severity.

Another limitation of this study is that the current approach of assessing exposure was conducted without the environmental distress of a pediatrician's office visit or the bias introduced through such settings. These findings do not account for interviewer bias or the Hawthorne effect, so this study likely overestimates the positive predictive value of the current approach to essential oil exposure.

4.2. Implications for Practice. The purpose of this study was to develop a reliable and valid instrument for use in epidemiological studies evaluating ingredients in personal care products. The APICHS was developed through a literature review and expert review. It was refined and finalized through a pilot test with retest and psychometric analysis.
Assessments of validity and reliability indicate that the APICHS provides substantial improvement to the current approach, dramatically reducing the risk of misclassification.

The approach is cost-effective, as there is no cost associated with a more thorough approach to identifying exposures of interest, but requires much more time than a single potentially biased question. The tool also requires regular updating to remain current with formulation changes and market trends, requiring clinicians to work with epidemiologists and environmental health specialists to ensure accurate classification of exposures.

\section{Conclusion}

This study provides evidence that the current approach of classification of exposure to aromatic plant ingredients poses a high risk of misclassification through both false positives and false negatives. In contrast, the APICHS was found to have exceptional sensitivity and specificity $(100 \%$ and $92.86 \%$, respectively) with positive predictive value of $97.22 \%$, far exceeding the sensitivity and specificity of the method currently in use.

Given the widespread use of essential oils in personal care and household products, the potential for endocrine disruption caused by these ingredients is a critical public health issue that needs to be urgently investigated. The APICHS is a valid, reliable tool for accurate classification of exposure to aromatic plant ingredients in personal care products and provides the tools necessary for this important research to be conducted.

\section{Data Availability}

The patient data used to support the findings of this study are restricted by Franklin Health's IRB in order to protect patient privacy. Data are available from Dr. Hawkins, j.hawkins@franklinhealth.org, for researchers who meet the criteria for access to confidential data.

\section{Conflicts of Interest}

The authors confirm they have no conflicts of interest to declare.

\section{Acknowledgments}

This study was funded by the Franklin Health Research Foundation, a nonprofit organization associated with the Franklin Health Research Center, which specializes in clinical and epidemiological research within the natural products industry. The Franklin Health Research Foundation is funded by generous donors, both corporate and private.

\section{References}

[1] D. Larson and S. E. Jacob, “Tea tree oil," Dermatitis: Contact, Atopic, Occupational, Drug, vol. 23, no. 1, pp. 48-49, 2012.

[2] P. A. Wu and W. D. James, "Lavender," Dermatitis: Contact, Atopic, Occupational, Drug, vol. 22, no. 6, pp. 344-347, 2011. 
[3] I. Arslan, S. Aydinoglu, and N. B. Karan, "Can lavender oil inhalation help to overcome dental anxiety and pain in children? a randomized clinical trial," European Journal of Pediatrics, vol. 179, no. 6, pp. 985-992, 2020.

[4] L. L. Barton, J. Carver, M. Szilagyi, S. Varma, C. D. Berkowitz, and E. M. Barksdale Jr., Advances in Pediatrics, E-Book 2018, Elsevier Health Sciences, USA, 2018.

[5] R. Soltani, S. Soheilipour, V. Hajhashemi, G. Asghari, M. Bagheri, and M. Molavi, "Evaluation of the effect of aromatherapy with lavender essential oil on post-tonsillectomy pain in pediatric patients: a randomized controlled trial," International Journal of Pediatric Otorhinolaryngology, vol. 77, no. 9, pp. 1579-1581, 2013.

[6] M. Ridder, "Essential oils market worldwide - statistics \& facts. Statista," 2020, https://www.statista.com/topics/5174/ essential-oils/.

[7] J. Hawkins, C. Hires, E. Dunne, and C. Baker, "The relationship between lavender and tea tree essential oils and pediatric endocrine disorders: a systematic review of the literature," Complementary Therapies in Medicine, vol. 49, Article ID 102288, 2020.

[8] V. López, B. Nielsen, M. Solas, M. J. Ramírez, and A. K. Jäger, "Exploring pharmacological mechanisms of lavender (Lavandula angustifolia) essential oil on central nervous system targets," Frontiers in Pharmacology, vol. 8, p. 280, 2017.

[9] G. Woronuk, Z. Demissie, M. Rheault, and S. Mahmoud, "Biosynthesis and therapeutic properties of Lavandula essential oil constituents," Planta Medica, vol. 77, no. 1, pp. 7-15, 2011.

[10] D. Bickers, P. Calow, H. Greim et al., "A toxicologic and dermatologic assessment of linalool and related esters when used as fragrance ingredients," Food and Chemical Toxicology, vol. 41, no. 7, pp. 919-942, 2003.

[11] W. Jāger, B. Našel, C. Našel et al., "Pharmacokinetic studies of the fragrance compound 1,8-cineol in humans during inhalation," Chemical Senses, vol. 21, no. 4, pp. 477-480, 1996.

[12] D. A. Grimes and K. F. Schulz, "Bias and causal associations in observational research," The Lancet, vol. 359, no. 9302, pp. 248-252, 2002.

[13] T. R. Vetter and E. J. Mascha, "Bias, confounding, and interaction: lions and tigers, and bears, oh my!" Anesthesia \& Analgesia, vol. 125, no. 3, pp. 1042-1048, 2017.

[14] S. Ackermann, F. Hartmann, A. Papassotiropoulos, D. J.-F. De Quervain, and B. Rasch, "Associations between basal cortisol levels and memory retrieval in healthy young individuals," Journal of Cognitive Neuroscience, vol. 25, no. 11, pp. 1896-1907, 2013.

[15] N. Golfenshtein, E. Srulovici, and B. Medoff-Cooper, "Investigating parenting stress across pediatric health conditionsa systematic review," Comprehensive Child and Adolescent Nursing, vol. 39, no. 1, pp. 41-79, 2016.

[16] A. Ganeshpurkar, R. Maheshwari, M. Tekade, and R. K. Tekade, "Concepts of hypothesis testing and types of errors," in Dosage Form Design Parameters, pp. 257-280, Academic Press, Cambridge, MA, USA, 2018.

[17] C. Cook, "Mode of administration bias," Journal of Manual \& Manipulative Therapy, vol. 18, no. 2, pp. 61-63, 2010.

[18] M. K. Salazar, "Interviewer bias: how it affects survey research," AAOHN Journal, vol. 38, no. 12, pp. 567-572, 1990.

[19] G. Tripepi, K. J. Jager, F. W. Dekker, and C. Zoccali, "Selection bias and information bias in clinical research," Nephron Clinical Practice, vol. 115, no. 2, pp. c94-c99, 2010.
[20] Q. Chen, H. Galfalvy, and N. Duan, "Effects of disease misclassification on exposure-disease association," American Journal of Public Health, vol. 103, no. 5, pp. e67-e73, 2013.

[21] J. T. Ramsey, Y. Li, Y. Arao et al., "Lavender products associated with premature the larche and prepubertal gynecomastia: case reports and endocrine-disrupting chemical activities," Journal of Clinical Endocrinology \& Metabolism, vol. 104, no. 11, pp. 5393-5405, 2019.

[22] T. F. Brady, T. Konkle, and G. A. Alvarez, "A review of visual memory capacity: beyond individual items and toward structured representations," Journal of Vision, vol. 11, no. 5, p. 4, 2011.

[23] K. Lindner, G. Blosser, and K. Cunigan, "Visual versus auditory learning and memory recall performance on shortterm versus long-term tests," Modern Psychological Studies, vol. 15 , no. 1 , p. $6,2009$.

[24] C. Stangor and J. Walinga, "How we remember: cues to improving memory," Introduction to Psychology, B.C. Open Textbook Project, Burnaby, Canada, 2019.

[25] US Food and Drug Administration, "FDA authority over cosmetics: how cosmetics are not FDA-approved, but are FDAregulated," 2005, https://www.fda.gov/cosmetics/cosmetics-lawsregulations/fda-authority-over-cosmetics-how-cosmetics-are-notfda-approved-are-fda-regulated.

[26] A. Berchtold, "Test-retest: agreement or reliability?" Methodological Innovations, vol. 9, Article ID 2059799116672875, 2016.

[27] L. I. Lin, "A concordance correlation coefficient to evaluate reproducibility," Biometrics, vol. 45, no. 1, pp. 255-268, 1989.

[28] J. Martin Bland and D. Altman, "Statistical methods for assessing agreement between two methods of clinical measurement," The Lancet, vol. 327, no. 8476, pp. 307-310, 1986. 